\title{
El modelo emprendedor de políticas culturales y el destination branding: un análisis a partir del filme Vicky Cristina Barcelona
}

Mariano-Martín Zamorano. Centro de Estudios sobre Cultura, Política y Sociedad, Barcelona, España.

RESUMEN | La literatura sobre la gobernanza de la política cultural de Barcelona ha revelado distintas tensiones políticas y sociales derivadas del uso de la cultura local con fines de creación de marca ciudad. No obstante, dichos estudios no se han centrado en las racionalidades detrás de este proceso ni en sus implicaciones para los modelos de la política cultural local en el ámbito cinematográfico. Con el objetivo de avanzar en el entendimiento de los modos de relación entre marca y política cultural, en este artículo analizamos una de las estrategias de cultural branding desarrolladas por la ciudad: la actividad pública en torno al filme Vicky Cristina Barcelona. Mediante el análisis de las relaciones políticas y los objetivos detrás de dicho proyecto, este artículo revela la instrumentalización gubernamental del patrimonio cultural de la ciudad con el fin de fortalecer y promover una marca global orientada al turismo.

PALABRAS CLAVE | política urbana, cultura urbana, marketing urbano.

ABSTRACT | The literature on the governance of cultural policy in Barcelona has revealed different political and social tensions arising from the use of local culture for the purpose of creating a city brand. However, these studies have not focused on the rationales behind this process nor on its implications for the models of local cultural policy in the cinematographic field. With the aim of advancing in the understanding of the modes of relationship between brand and cultural policy, in this article we analyze one of the cultural branding strategies developed by the city: the public activity around the film Vicky Cristina Barcelona. Through the analysis of political relations and the objectives behind this project, this article reveals the governmental instrumentalization of the city's cultural heritage in order to strengthen and promote a global tourism-oriented brand.

KEYwORDs | urban policy, urban culture, urban marketing. 


\section{Introducción}

Diversas transformaciones sociales, económicas y políticas ocurridas desde los años ochenta dieron paso a un nuevo orden geopolítico, donde la política cultural sufrió múltiples mutaciones. La globalización económica y tecnológica y la cristalización del modelo de Estado posfordista (Jessop, 2002), otorgaron mayor centralidad a la economía de la cultura en los países desarrollados. Por otra parte, este desarrollo se interrelacionó con la emergencia de las llamadas sociedades posindustriales, donde la producción y el consumo de las artes y la cultura adquirieron una gran significancia (Bell, 1976; Rodríguez Morató, 2007). Dichos procesos contribuyeron a la diversificación de los ámbitos de actuación de la gestión pública de la cultura, así como a brindarle una renovada trascendencia internacional.

En este escenario, las ciudades se transformaron en protagonistas de las políticas culturales, y sus gobiernos agruparon recursos y adquirieron nuevas competencias en este ámbito (Bianchini, 1993). De hecho, paralelamente al cuestionamiento del dominio de los gobiernos centrales en la política cultural (Menger, 2010), las instituciones culturales subestatales y supraestatales cobraron mayor capacidad de acción en dicho campo. En este marco, tanto las ciudades como las regiones intensificaron su acción cultural exterior, persiguiendo establecerse como centros de incidencia internacional (Négrier, 1997). Uno de los efectos particulares de esta transformación ha sido la progresiva imbricación de la política cultural local -particularmente de aquella desarrollada por las grandes ciudades- con la diplomacia cultural (Zamorano \& Rodríguez Morató, 2015). Esta dinámica de internacionalización de la política cultural urbana coincidió, desde los años ochenta, con su llamado "giro emprendedor" en el mundo desarrollado (Bianchini, 1993; Harvey, 1989). Además, los nuevos discursos que enmarcaron esta actividad, como la creatividad o la competitividad, reafirmaron el carácter transversal y sistémico de la dimensión internacional en la gestión de las artes y la cultura. Esta nueva fase de internacionalización de la política cultural urbana fue, por lo tanto, coincidente con un replanteamiento de su gobernanza público-privada (Peters, 1995), ahora caracterizada por nuevas alianzas públicas con distintos sectores de servicios, como la industria turística (Benach \& Tello, 2004).

Por otra parte, en los años noventa, la hegemonía del modelo económico neoliberal a nivel mundial profundizó la tensión entre los intereses del mercado transnacional y los intereses soberanos de los países (Cox, 1999). En línea con dicha tendencia, la política cultural exterior mutó su lógica de actuación y se inscribió en el paso del Estado regulador al Estado competitivo, para el cual el éxito comercial (del capital nacional) en el mercado internacional es un criterio de actuación central (Opello \& Rosow, 1999). Esta renovada relación entre sistema internacional y soberanía estatal forjó la adecuación de la acción cultural exterior al modelo instrumental que favorece la globalización de la economía. Así, la utilización de la cultura como instrumento de creación de marca-lugar, se situó como una rationale fundamental de la política cultural doméstica y exterior, y como un mecanismo de disputa en el escenario internacional (Aronczyk, 2008; Villanueva, 2007). Este proceso se vio acompañado por una transformación del modelo histórico de Estado monopolista 
en la acción cultural exterior (diplomacia de la cultura), caracterizado por su orientación nacionalista y monocultural. Dicha perspectiva dio lugar a la extensión de distintas estrategias mayormente centradas en la "competitividad cultural" de la acción cultural internacional. Este conjunto de trasformaciones favoreció la sistematización de la acción cultural exterior de las ciudades (la llamada paradiplomacia ${ }^{1}$ cultural), donde la proyección internacional de las marcas-lugar y el uso de la industria cultural para su fortalecimiento se fueron situando como ejes de actuación fundamental (Hutson \& Brent Ritchie, 2006; Zamorano \& Morató, 2015).

En el presente artículo analizaremos cómo se ha desarrollado la marca-lugar orientada al turismo para comprender qué transformaciones supone en relación con el diseño y la implementación de la política cultural local. Esta problemática ha sido estudiada durante las últimas décadas, particularmente teniendo como objeto de análisis el proyecto emprendedor de la ciudad creativa (Florida, 2002), donde los grandes eventos y la creación de clústeres culturales -con un acento en los medios digitales- se presentan como estrategias de posicionamiento global (García, 2004; Hutton, 2017; Majoor, 2011; Rius Uldemollins, 2014). No obstante, pese a la reproducción del modelo de ciudad creativa a nivel mundial y a la intensificación de sus estrategias dedicadas a la atracción de turistas, la relación entre destination branding y política cultural, tanto en términos de representatividad política como de modificación de las pautas de acción de la gestión local de la cultura, no ha sido estudiada en profundidad. Este fenómeno tampoco ha sido analizado para el caso de las políticas culturales cinematográficas. Por lo tanto, la racionalidad que supone este modelo y sus implicaciones para las políticas públicas - particularmente para aquellas orientadas al cine- deben ser abordadas.

El presente estudio de caso, centrado en las políticas públicas en torno al filme Vicky Cristina Barcelona, se basa en una metodología cualitativa que abarcó la realización de trabajo de campo. Además de un importante trabajo de escritorio, entre 2013 y 2014 se realizaron 31 entrevistas con agentes relevantes correspondientes a las instituciones públicas de tres niveles de gobierno, responsables de empresas representativas de los distintos sectores artísticos catalanes -incluido el audiovisual-, y otros actores sociales en el ámbito de la acción cultural internacional (véase ficha completa en tabla 1 anexa). Se trató de entrevistas semiestructuradas con informantes clave, que persiguieron identificar los factores que contribuyeron al establecimiento y transformación de los distintos modelos de la gobernanza cultural exterior de la ciudad de Barcelona y de sus mecanismos de proyección internacional. Las variables de análisis, reflejadas en el esquema del guion de cada entrevista, se pueden agrupar en tres ejes: a) el institucional, donde analizaron los discursos legitimadores y objetivos de cada organización a la hora de diseñar o implementar la política cultural exterior y de marca regional y urbana; b) el de interacciones y relaciones (público-privadas-asociativas, para cada caso), donde se trataron los modos de vinculación intergubernamental y de la administraciones de Barcelona y Cataluña con otros actores; y finalmente c) el histórico, donde se abordó la evolución general

El concepto de paradiplomacia hace referencia a aquellas actividades diplomáticas desarrolladas por entidades subestatales. 
de la paradiplomacia cultural de Barcelona y Cataluña. El análisis del fenómeno cinematográfico formó parte de cada dimensión de estudio. En particular, se persiguió contrastar cómo el audiovisual fue incorporado a la política cultural exterior local y regional, teniendo en cuenta sus distintas orientaciones.

Sobre esta base, el presente artículo examina la influencia de las políticas de internacionalización de la imagen urbana sobre los marcos de actuación de la política cultural actual. Mediante el análisis de las políticas culturales audiovisuales de la ciudad y de la Generalitat catalana en torno al filme Vicky Cristina Barcelona (2007), se identificarán los distintos objetivos detrás de su diseño e implementación. Este análisis persigue, de este modo, contribuir a singularizar el rol del cine dirigido al turismo en las políticas culturales de orientación emprendedora. La hipótesis del artículo plantea la existencia de un proceso de instrumentalización de los bienes culturales que tiene por objetivo aumentar la eficiencia turística de las políticas de branding, sobre la base del potencial de la industria cultural y, en este caso, de la asociación de un director de renombre como Woody Allen con la marca Barcelona.

El texto comienza definiendo place branding y acotando sus especificidades en el ámbito de la política cultural mediante un estudio de la literatura en este campo. En el siguiente apartado se analiza la transformación de las políticas culturales centradas en el branding, en términos de internacionalización de esta actividad y de reorientación de sus objetivos y prioridades. En tercer lugar, se desarrolla una acabada descripción de las políticas en torno a la película Vicky Cristina Barcelona y se consideran sus discursos legitimadores. Finalmente, se realiza un análisis del caso en las conclusiones. A partir de este caso, consideraremos cómo frecuentemente el branding y la internacionalización de los sectores culturales suponen aproximaciones divergentes - pero de límites difusos- a la "definición" y gestión de las identidades colectivas por parte de los actores gubernamentales (Mark, 2008, p. 13). La demarcación de ambas esferas conceptuales y políticas, así como el estudio de sus específicos modos de interrelación, son muy relevantes para avanzar en la comprensión de la política cultural actual, donde la búsqueda de externalidades internacionales como el turismo posee una creciente influencia.

\section{Place branding y políticas públicas en el ámbito cultural}

El concepto de branding define originalmente los diferentes métodos publicitarios destinados a favorecer la asociación mental entre un producto y un nombre o una imagen específicos, así como a dotar a dicho producto de reputación y valor simbólico. En el mundo contemporáneo, este mecanismo de mercantilización se ha extendido a múltiples esferas sociales, que van desde las personas (personal branding) hasta los lugares. El sistema de evaluación de "imagen-lugar" desarrollado durante las últimas décadas, se ha definido como el procedimiento necesario y previo al diseño del márquetin territorial (Anholt, 2006). A partir de la información recabada sobre la percepción social exterior de un determinado espacio sociopolítico, se aplican técnicas de marca corporativa por parte de agencias y departamentos de diversos gobiernos, y de empresas nacionales e internacionales de estudio de 
mercado, con el fin de promover la asociación externa entre lugar e imagen y de mejorar dicha imagen (Dinnie, 2008).

Desde fines de los años noventa, el despliegue de la llamada "ciudad creativa" ha sido uno de los proyectos centrales en la promoción y legitimación de las marcas urbanas por parte de las administraciones públicas. Dicho modelo se inscribe en el contexto de la voluntad de revitalización de los entornos urbanos posindustriales, entonces económicamente deprimidos. La generación de la "ciudad creativa" supuso un nuevo modelo de articulación entre actores públicos y privados, y sus estrategias de proyección global persiguieron la atracción de recursos financieros internacionales y de profesionales altamente educados y capacitados: la denominada "clase creativa" (Florida, 2002). El desarrollo de políticas públicas orientadas al establecimiento de entornos adecuados para la vida y el trabajo de dicha "clase creativa" se ha enmarcado en el llamado entrepreneurial turn de las políticas locales (Harvey, 1989). Los mecanismos distintivos que articularon esta transformación han sido los proyectos de revitalización urbana sobre la base de grandes planes arquitectónicos e instituciones culturales (Bianchini, 1993), los eventos espectaculares (García, 2004) y la creación de clústeres de industrias culturales (Scott, 2010).

Se ha señalado que estas políticas han buscado la promoción de aquellas condiciones infraestructurales y sociales que permiten a una ciudad alcanzar el estatus de "creativa" (Vanolo, 2008). Esta política estuvo basada tanto en la transformación del entorno local, como en la organización de su representación exterior en tanto espacio renovador y dinámico. Es decir, que un objetivo común de estas estrategias ha sido la generación de una imagen de ciudad innovadora (Landry \& Bianchini, 1995), teniendo en la cultura un instrumento central de transformación económica y de márquetin (Zukin, 1996). Todo esto implicó cambios sustanciales en la racionalidad aplicada al diseño e implementación de las políticas públicas (Vicari Haddock, 2010). Entendemos como racionalidad de las políticas públicas el marco lógico e ideológico que justifica la selección de una opción o programa políticos entre una serie de opciones posibles -y legales- (Schneider \& Ingram 1997, p. 2). En el proyecto de la ciudad creativa, la búsqueda de atracción de capitales y recursos humanos mediante técnicas de marca cultural se situó como requisito para el desarrollo local, y dichos objetivos fueron frecuentemente priorizados frente a políticas alternativas.

De acuerdo a estas premisas, los proyectos de city branding se han replicado en las grandes capitales mundiales, y también en las ciudades medianas que pretenden desarrollar una estrategia propia para competir en la economía global. De esta manera, se ha buscado instaurar una marca de ciudad que consiste en la promoción holística de la ciudad a partir de su asociación con un icono cultural global (Evans, 2003). Uno de los objetivos de las políticas urbanas y de las políticas culturales ha sido promover y destacar los elementos "auténticos" de cada territorio para articular una estrategia de place branding (Jones \& Smith, 2005).

La lógica en la que se inscriben los sistemas de evaluación y diseño de marca lugar (Anholt, 2006) se basa en la posibilidad de una "rectificación" coherente -es decir, con la mayor coordinación y sintonía posible de todos los sectores implicadosde aquellos aspectos que perjudican su imagen en función de una configuración 
competitiva de la misma. En este contexto, la representación de la cultura local o nacional se figuran como un capital simbólico más, pregnante e informativo que, una vez difundido, otorga unas determinadas cualidades positivas a un territorio. Uno de los registros centrales de este branding territorial es el destination branding, mayormente orientado a fomentar la industria turística mediante la asociación del lugar con productos y servicios de ocio (Moilanen \& Rainisto, 2008, p. 112). Esta forma de branding se caracteriza por enfatizar el "nombre, símbolo, logotipo, palabra u otro gráfico que identifique y diferencie el destino (...) mientras trasmite la promesa de una experiencia de viaje memorable" (Hanna \& Rowley, 2008, p. 63). Promocionada mediante estas estrategias, la ciudad deviene en un potencial espacio de consumo y ocio, pero también en un capital simbólico que puede permitir disponer de un cierto estatus a su consumidor. El turista, en las distintas experiencias que supone su relación con la ciudad visitada, es su propio objeto de marca, en la medida en que es capaz de impresionar a otros mediante la exposición pública de su visita, en línea con las ideas de Veblen (1994) sobre la clase ociosa, y de Bourdieu (2003, p. 75) sobre la relación entre fotografía turística y poder simbólico.

\section{Las estrategias de marca en la acción cultural exterior y sus implicaciones domésticas e internacionales}

Dado que la cultura de un lugar es considerada uno de los factores fundamentales en la generación de marca, los límites conceptuales y prácticos entre el branding y la política cultural o la diplomacia cultural se manifiestan frecuentemente difusos (Mark, 2008, p. 60; Villanueva, 2007; Zamorano, 2016). En este sentido, bajo el criterio instrumental de la competencia en el sistema internacional, las estrategias de marca asumen cada vez más importancia y se disputan espacios de actuación con la política cultural exterior (Rius Ulldemolins \& Zamorano, 2015). Por lo tanto, un factor clave en el análisis y desarrollo de la política cultural actual es la relación entre cultura y branding.

La acción cultural exterior adopta frecuentemente una orientación de branding basada en una racionalidad que persigue la generación de familiaridad y de apreciación exteriores sobre una nación o un territorio. Y, siendo el branding una actividad cuyo objetivo central y modelador es alcanzar una mejor posición en el mercado capitalista, su fuente de legitimidad suele asociarse a las fuerzas del mercado (Villanueva, 2007, p. 25). Esto orienta el diagramado de la política cultural hacia técnicas de diseño (específico y medible) centradas en la capacidad de persuasión de los contenidos, es decir, en la visibilización de los "beneficios del producto".

Desde esta perspectiva, el ciudadano en el exterior es visto mayormente como consumidor y el público local como "soporte" de la marca. Por lo tanto, se ha destacado que, para el éxito de esta estrategia, es fundamental que los ciudadanos "vivan la marca”, es decir, que se identifiquen y participen de esta imagen de cara al exterior (Anholt, 2003, p. 123). Se trata, así, de una dinámica siempre conceptuada como posterior a la existencia de la marca que los representa, a partir de la cual el branding dota de un nuevo uso a los llamados "imaginarios espaciales" (Lewis, 2011, p. 264), orientando estas representaciones en función del desarrollo económico de sectores 
privados transnacionales o de la promoción externa e interna de formas identitarias. En este sentido, el place branding y la política exterior en cultura poseen en común un componente de construcción de identidad; es decir, se constituyen en distintas representaciones que promueven un imaginario común en la sociedad representada (Anholt, 2003, p. 123; Widler, 2007).

Pero la "pregnancia" de la imagen cultural basada en esta síntesis favorecería distintas distorsiones respecto de su objeto en tanto diverso, limitando los registros redistributivos y representativos de la política cultural. Así, mientras en el marco del pensamiento y de la literatura sobre márquetin y economía en torno al place branding esta actividad se inscribe en la esfera de una moral globalizante en torno a la creatividad y la libre elección y expresión individuales, en los campos de la sociología y de la geografía se han desarrollado lecturas críticas sobre el tema, orientadas a identificar los modos de construcción de las marcas y sus efectos en la sociedad (Lewis, 2011, p. 267). Estos estudios han advertido que la implementación del márquetin país ha tenido diversas consecuencias sociales y políticas negativas. Tempranamente, en 1997, la iniciativa política laborista de nation branding fue criticada como un intento de convertir Gran Bretańa en un producto comercial (Leonard, 1997). En cuanto a la representación en sí misma, se ha advertido sobre el riesgo de construir imágenes banales y repetitivas (Evans, 2003; Ren \& Blichfeldt, 2011). Se ha manifestado cómo estas estrategias comportan frecuentemente la implementación de políticas elitistas y gentrificadoras (Balibrea, 2017; Delgado, 2008; Peck, 2005), y que las políticas culturales se han visto crecientemente instrumentalizadas política y económicamente con el fin de fortalecer la marca territorial mediante las industrias culturales (Dinardi, 2017; Volkerling, 2001). En la misma dirección, también se ha indicado que la aplicación de estos principios supone en muchos casos la privatización de una función pública - dada su frecuente delegación a consultoras privadas- (Aronczyk, 2008), y que vincula, desde una perspectiva de difusión unidireccional o "de flujo de mensajes en un solo sentido" (Leonard, 2002, p. 48) a un territorio con el exterior.

La publicidad de las ciudades mediante la cinematografía ha sido un instrumento de marca a lo largo de la historia del séptimo arte, por ejemplo, con filmes como Roman Holiday. La película, dirigida por William Wyler y protagonizada por Audrey Hepburn (1953), sirvió como un eficaz instrumento de promoción de la capital italiana en el mundo anglosajón (Hernández Ramírez, 2004). Esta presentación de las geografías mediante su ficcionalización cinematográfica ha demostrado ser medio de impulso y atracción de personas y capitales hacia las locaciones de los filmes (Stanishevski, 2007; Urry, 2002). Se ha considerado que el cine posee un gran poder como inductor del turismo, dada su capacidad de persuasión sobre la imaginación de los espectadores, condicionando de este modo la elección de los destinos (Del Rey-Reguillo, 2007). Así, sobre la base del gran potencial propagandístico de la industria cultural, desde los ańos noventa han ido ganando terreno políticas públicas de creación de marca ciudad basadas en el cine (Chen \& Mele, 2016), así como políticas orientadas a posicionar la marca cinematográfica nacional (Falicov, 2018, p. 379). Las mismas tienen como un objetivo principal el incremento del turismo, pero, en el marco de las políticas culturales, presentan también otros 
objetivos, como la estimulación de la producción cinematográfica local. Dichos fines se articulan mediante herramientas diversas (como, por ejemplo, la labor de las Film Commissions) y hacen navegar estas estrategias entre el branding y la política cultural exterior.

\section{Vicky Cristina Barcelona como política pública}

Vicky Cristina Barcelona fue dirigida por el cineasta estadounidense Woody Allen y rodada en distintas ciudades de Nueva York, Cataluña y Asturias durante el año 2007. Cabe señalar que el vínculo del director con la política cultural internacional de Cataluña se estableció con anterioridad. En el año 2001, Allen participó en la realización de la "Semana de la Cultura Catalana" en Nueva York tras una invitación del gobierno catalán. El entonces director general de Promoción Cultural del Departamento de Cultura señala al respecto:

Pero aquel Consejero [el entonces Conseller de Cultura catalán, Jordi Vilajoana] estuvo muy hábil, porque consiguió un padrinazgo muy potente que es el de Woody Allen, con unos medios absolutamente mínimos. El vino a un acto que hicimos en Nueva York y conseguimos que vaya toda la prensa (...) estuvo nada, un momento, habló, estuvimos con él. Intentamos todos hacernos una foto todos con Woody Allen y conseguimos un impacto brutal aquí. Cuando digo aquí, digo en toda España, porque fue toda la prensa hispana en Nueva York. (Llorca, entrevista personal, 13 de diciembre de 2013)

El proyecto de Vicky Cristina Barcelona tuvo origen dos ańos después de dicho evento, cuando Woody Allen reveló su intención de filmar en España. Entonces la productora de capital catalán Mediapro $^{2}$ estableció negociaciones con Allen, con la intención de que el filme proyectado por el director se rodara en la ciudad de Barcelona y con actores españoles como protagonistas. Por otra parte, la Fundación Príncipe de Asturias persuadió al cineasta para que Asturias asumiera también un espacio en la película (Aertsen, 2011, p. 5) y de este modo acompańar la política de marca del Principado (Martínez Expósito, 2014, p. 348). Con la producción avanzada, en marzo de 2007, el entonces alcalde de la ciudad de Barcelona, Jordi Hereu, tuvo un encuentro con el director en Nueva York en el marco de un viaje de Gobierno a dicha ciudad. En el mismo se discutieron aspectos de la gestión del filme (Celis, 2007).

Como producto de estas relaciones, la película fue coproducida por empresas privadas estadounidenses y españolas, y contó con un amplio apoyo público. Se trató de una coproducción igualitaria donde participaron, por los Estados Unidos, Gravier Productions -que produjo los últimos largometrajes de Woody Allen- y, por España, la empresa catalana Mediapro, con la producción asociada de Antena 3 Films, Antena 3 Televisión y Televisió de Catalunya (тv3) (Confederación de Productores Audiovisuales Españoles [FapaE], 2008, p. 40).

Megaproducción sL es una empresa con sede central en Barcelona dedicada a la edición, grabación y producción videográfica de obras culturales, deportivas y artísticas. 
El proyecto recibió ayudas por parte del Ayuntamiento de Barcelona, que aportó 1 millón de euros, ${ }^{3}$ y de la Generalitat de Cataluña, que contribuyó con otros cerca de 500 mil euros; 199.924,83 euros del Departament de Cultura i Mitjans de Comunicació para la empresa Mediapro (Generalitat de Catalunya, 2010) y el resto correspondió a la Consejería de Innovación, universidades y empresas. Particularmente, la contribución del Ayuntamiento de Barcelona se concretó mediante la empresa de capital riesgo BCN Emprèn y se estipuló el retorno en función de la recaudación del filme. De esta manera, el consistorio, en manos del Partido Socialista de Cataluńa, se constituyó en un promotor fundamental de la película, un hecho que fue cuestionado por la oposición política y destacado por la prensa internacional (Pingree, 2007). Este millón y medio de euros de aportes públicos representó alrededor del 10\% de los aproximadamente 15,5 millones de dólares que se invirtieron para llevar adelante la producción (Internet Movie Database, IMDb). Cabe señalar que también se firmó un contrato entre el consorcio público Turisme de Catalunya y la productora Mediapro, que estipuló un patrocinio de 750.000 euros, con el objetivo de "aprofitar el gran impacte mediàtic i comunicatiu que el Sr. Allen té internacionalment i, en particular a Europa, mercat de procedencia de la gran majoria del turisme estranger amb destinació Catalunya." (Parlament de Catalunya, 2008, p. 66). A cambio, se estipuló rodar la mayoría de las escenas de la película en Cataluña e incorporar el logo de Turisme de Catalunya en los créditos y otras contribuciones de promoción de la película. Finalmente, la película contó con el apoyo del Ministerio de Cultura español mediante la amortización directa en taquilla, un acuerdo que significaba un aporte del 15\% de la recaudación de la película, con la condición de que esta superara los 300 mil espectadores (Agencias, 2007). ${ }^{4}$

Esta alianza de la producción de Woody Allen con el capital privado y público español permitió al director relanzar su carrera, entonces dificultada por el escaso éxito comercial de sus películas en los Estados Unidos. ${ }^{5}$ El impulso político y económico a dicha producción por parte de las distintas administraciones públicas del Estado español fue establecido prioritariamente desde una lógica vinculada a sus potenciales beneficios para la difusión de Barcelona, Cataluña y España en el mundo anglosajón. Así lo indicaba Toni Puig, consultor turístico de Barcelona, quien señalaba cómo el apoyo a esta política se consideró en función de la necesidad de situar la marca de la ciudad entre el público estadunidense (Rodríguez Campo, Fraiz Brea, \& Rodríguez-Toubes Muñiz, 2011, p. 148). En esta línea, con la mediación de las administraciones implicadas, se le requirió también al director estadounidense la aparición del término 'Barcelona’ en el título de la película.

3 Véase la promoción institucional del filme en https://www.barcelonaturisme.com/updates/20/ update_cat_print.html

4 Como parte de esta promoción pública, la empresa Mediapro recibió 4750 euros por parte del Instituto Catalán de Industrias Culturales (ICIC), destinados a apoyar la presentación del filme en el Festival de Cannes (ICIC, 2008, p. 57).

5 El acuerdo de coproducción entre Allen y Mediapro abarcó tres filmes que fueron un éxito de recaudación. En el caso de Midnight in Paris, la segunda de la tríada, se trató de la película de mayor éxito comercial en la historia del cineasta. 
La trama del filme y el modelo de proyección internacional de la ciudad

La descripción y la construcción romántica del entorno urbano han sido algunas de las claves de la estética cinematográfica de Woody Allen (Bailey, 2001, p. 54). Sin embargo, a diferencia de sus clásicas referencias intimistas y de tono psicológico sobre la ciudad de Nueva York, en Vicky Cristina Barcelona "Barcelona es enfocada a través de la mirada esquematizada del turista” (Martínez Expósito, 2014, p. 352). La narración del filme se vertebra en torno a la experiencia turística de sus protagonistas en el entorno urbano, desde una mirada exotizante. ${ }^{6}$ El relato se traduce en una suerte de campańa publicitaria de la ciudad, debido a su recurrencia en los activos patrimoniales globales y los tópicos culturales. La visión cultural, artística y bohemia de la urbe se manifiesta en varios planos a lo largo de la historia; por ejemplo, mediante el uso constante del flamenco como banda de sonido y la asistencia a conciertos de este género musical por parte de los personajes. Además, la historia tiene como escenografía un recorrido visual por los barrios característicos de Barcelona y cuenta con múltiples escenas ambientadas en las locaciones más representativas del patrimonio urbano (arquitectónico y paisajístico) de la ciudad, apareciendo de manera consecutiva: 1) Mosaico de Miró (Terminal 2 Aeropuerto El Prat); 2) Iglesia de la Sagrada Familia (dos escenas, de frente y como fondo); 3) Museo Miró (recorrido); 4) La Pedrera (dos escenas, terraza y frente); 5) Bar Marsella (tres escenas, dos interior y una exterior); 6) Parque Güell (dos escenas); 7) Parque Guinardó (con vistas a la ciudad); 8) Parque de la Ciutadella; 9- Hotel Casa Fuster; 10) Mural de Chillida y Museo de Arte Contemporáneo de Barcelona (мAсвA) (Raval); 11) Ramblas (dos escenas); 12) Museo Nacional de Arte de Cataluña (MNAC) (y escaleras del Montjuic); 13) Pabellones Güell (Pedralbes).

Por su parte, Woody Allen señaló que había plasmado en el guion su propia percepción sobre la ciudad, del mismo modo que lo había hecho con Manhattan a lo largo de su carrera, "muy a través de mis ojos" (Fresán, 2012). De este modo, parece darse un cierto ensimismamiento entre la historia turística que incorpora el relato y el enfoque estético de dicha experiencia que propone el autor.

En esta línea, el contenido de la película fue cuestionado en lo referente a su escaso reflejo de la realidad local. Así, mientras algunos medios de comunicación calificaron la película como un "publirreportaje turístico" (Montalt, 2007), desde una perspectiva antropológica se subrayó su falta de representación de la realidad lingüística y su carencia de autenticidad en relación con los modos de vida locales (DiGiacomo, 2008). En este sentido, la película se centra en la caracterización de Barcelona como una ciudad característicamente española, con alusiones recurrentes al flamenco y a la pasión de sus habitantes. En cambio, se hace una interpretación de la identidad catalana solo en clave patrimonial, vinculada a la peregrinación por sus rincones de consumo y recreo (Aertsen, 2011). Es decir, se apela a la iconografía internacional del territorio, a recursos como el verano, la gastronomía o las artes plásticas, pero haciendo alusiones secundarias a la lengua catalana o a sus factores diferenciales como cultura nacional.

6 La trama del filme se centra en un viaje de vacaciones de dos jóvenes estadounidenses, Vicky (Rebecca Hall) y Cristina (Scarlett Johansson), por distintas ciudades de España. Las protagonistas, de personalidades opuestas, conocen durante su itinerario a un artista espańol, Juan Antonio, encarnado por Javier Bardem. 


\section{Derivaciones económicas y políticas del filme}

En lo referente a la crítica y al público, el filme cosechó múltiples galardones, otorgándole una mayor notoriedad internacional. Penélope Cruz, quien actuó en un papel secundario, obtuvo un premio Oscar y otros diversos galardones. En esta línea, el filme tuvo una gran repercusión mediática en la prensa internacional, que en algunos casos enfatizó diversas facetas de su relación con la ciudad y con su producción (Dargis, 2008). La película fue también un éxito comercial y catapultó tanto a Woody Allen como a la productora Mediapro. Tuvo una gran recaudación de taquilla en diversos países: recogió, solo en Cataluña, 2,5 millones de euros el primer año (2008). Fue uno de los diez largometrajes más vistos en la región durante ese año, la única de esta decena que contó con producción nacional y la más vista del año en lengua catalana (Departament de Cultura, 2008). A nivel del conjunto del Estado español recaudó, en el mismo año de su lanzamiento, 7,5 millones de euros y contó con un público de 1,2 millones de personas (Ministerio de Cultura, 2008). En el terreno internacional también fue un éxito comercial, con una gran asistencia de público en Estados Unidos, llegando a ingresar casi siete veces su inversión, con una recaudación total estimada, ${ }^{7}$ hasta 2011, de 96,4 millones de dólares (Menchén, 2011).

Por otro lado, se encuentran aquellos elementos vinculados a la participación pública en la producción del filme. En lo referente al Ayuntamiento de Barcelona, el lanzamiento coincidió con un cierto estancamiento relativo de la demanda turística que presentaba la ciudad: en 2007, había superado los 7 millones de visitantes (Turisme de Barcelona, 2010, p. 7). Este proceso derivaría en un leve descenso tras el año 2009, en el marco de la crisis económica internacional, lo que a posteriori de la realización de la película otorgaba una mayor legitimidad a las políticas orientadas a la potenciación del turismo anglosajón. Además, el apoyo por parte del Ayuntamiento puede ser considerado un éxito desde la perspectiva del consumo del filme y de su capacidad de posicionamiento de la marca ciudad. En este sentido, una encuesta al público gallego evidencia que la película tuvo un impacto significativo en la transformación de las representaciones de los espectadores sobre la ciudad, a la vez que manifestaba una cierta capacidad en la generación de atracción de personas (Rodríguez Campo et al., 2011, p. 153). ${ }^{8}$

Otro registro de la intervención de la administración local en la producción estuvo relacionado con la aportación de la película a los sectores productivos asociados a la industria local del cine. El rodaje, que contó con la colaboración de la Barcelona-Catalunya Film Commission, era interpretado por el Instituto de Cultura de Barcelona (ICUB) como parte de la proyección de la imagen de la ciudad y de su posible potenciación como plató:

$7 \quad$ Al 8 de marzo de 2009, Vicky Cristina Barcelona había recaudado 91.262.911 millones de dólares en todo el mundo (Box Office Mojo. https://www.boxofficemojo.com/movies/?id=vickycristinab arcelona.htm).

8 La administración local desarrolló también diversas iniciativas, como, por ejemplo, el programa de la oficina de Turisme de Barcelona "Barcelona Movie Walks". Se trata de rutas por la ciudad a lo largo de distintas locaciones de películas con fama internacional, como Vicky Cristina Barcelona o Todo sobre mi madre, de Pedro Almodóvar (ICUB, 2008, p. 241). 
Pero, más allá de las cifras, es evidente que los rodajes que tienen más repercusión mediática para una ciudad son los largometrajes de cine, algunos de los cuales pueden llegar a diversos lugares del mundo. En 2007 Barcelona ha sido escenario de hasta 33 largometrajes para la gran pantalla. Es de obligado recordatorio, por ejemplo, el rodaje de la película de Woody Allen Vicky Cristina Barcelona, con Scarlett Johansson, Penélope Cruz y Javier Bardem, que ha recibido el apoyo de las administraciones públicas. (ICUB, 2007, p. 159)

No obstante, cabe señalar que las políticas culturales de desarrollo local del ICUв no siempre han estado en consonancia con las orientaciones propuestas por las áreas de Turismo y Promoción Económica del Ayuntamiento para la internacionalización cultural de la ciudad. En este sentido, el responsable de promoción cultural del ICUB, Esteve Caramés, indica:

Sobre todo en los últimos cinco años tenemos una tensión como Departamento de Cultura con el Departamento de Promoción Económica. Porque desde el Departamento de Promoción Económica también han encontrado que eso de la Barcelona creativa es una marca fantástica. De promoción turística, de promoción comercial y promoción económica de la ciudad. Ahora nos viene muy bien explicar esta fancy Barcelona, tal, no sé qué. Nosotros nos lo miramos lejano todo eso. Es decir, todo el mundo Richard Florida, nosotros desde aquí nos lo miramos lejano.

(Caramés, entrevista personal, 19 de septiembre de 2013)

En cambio, la participación de la Conselleria de Cultura catalana en la producción de la película se enmarcó en una lógica que tiene puntos comunes y otros disímiles a los propuestos por el Ayuntamiento. Se trató de una política que otorgaba particular importancia al impulso del sector cinematográfico en catalán y en Cataluña, desarrollada en el contexto del llamado Gobierno tripartito de izquierdas, y que agrupaba posiciones nacionalistas de izquierda, progresistas y socialistas (2006-2010). Dicha intervención del Gobierno autonómico fue calificada como intervencionista y propagandista por distintos agentes de la oposición al Gobierno tripartito, ubicados en un bloque liberal, como Ciudadanos (De Rivera, 2009) así como por parte del Grupo Mixto (Generalitat de Catalunya, 2008, p. 42).

La producción de la película se dio en el marco de distintos diagnósticos y transformaciones ocurridas en el área de la producción audiovisual de la Comunidad Autónoma. Por un lado, las coproducciones cinematográficas con participación de empresas catalanas continuaban siendo bajas. Asimismo, las películas en versión original en catalán llegaban al 1\% de cuota de mercado y recibían solo el 2,25\% de los espectadores (Moragas i Spa, 2009, p. 125). El creciente apoyo de la política cultural autonómica a estas propuestas se presentaba como un mecanismo de promoción del empresariado local y exterior en este sector a nivel regional. ${ }^{9}$ Desde el Instituto Catalán de Industrias Culturales (ICIC), Ferran Tomas, entonces a cargo del área audiovisual, mencionaba que el apoyo a la película buscaba "una mayor 
presencia de talentos locales y que fortalezca la participación catalana en coproducciones" (Montalt, 2007). Posteriormente se destacó el "efecto Woody Allen”, que habría favorecido el aumento de rodajes en la ciudad, que llegaron a sesenta en el año 2008, un 13,5\% más que el año anterior (ICUB, 2008, p. 224). Si bien el número de pelícuas producidas en Cataluña también creció entre 2007 y 2008 -de 121 a 137-, cabe considerar que el número de películas con participación de empresas con sede social en Cataluña decreció de 1034 películas a 928 en el mismo periodo (Instituto de Estadística de Cataluña [Idescat], 2010).

Por otra parte, la Conselleria de Cultura impulsó la exhibición del filme de Allen solo en catalán, en un acuerdo derivado de las ayudas públicas otorgadas. Como parte de esta iniciativa, inicialmente la productora Mediapro había previsto que su estreno se produjera solo en versión original y en catalán (Cazorla, 2008). Descartada esta opción tras la presión de las multinacionales estadounidenses al grupo empresarial, se optó por hacer prevalecer la distribución en catalán, con cincuenta copias en este idioma, cinco en inglés y cinco en castellano. A pesar de esta política, predominó su visualización en versión doblada en castellano. No obstante, fue el "largometraje más visto de los exhibidos en catalán" en 2008, con 140.765 espectadores (Moragas i Spa, 2009, p. 124). En la misma dirección, como sucedió con diversas películas, fue una oportunidad para articular un acuerdo para la difusión de la versión doblada al catalán del filme en el mercado local mediante la TV de Cataluña, Tv3 (Savall, 2009). En consecuencia, distintas medidas en torno a esta película formaron parte de un programa orientado a promover el cine en catalán y las coproducciones con capitales de la región, y pueden ser consideradas la antesala de la Ley de Cine catalana de 2010 (Savall, 2009). En estos términos, la película sirvió relativamente al desarrollo de la industria audiovisual regional, principalmente en lo referido a rodajes e inversión exterior.

\section{Conclusiones}

En este artículo hemos perseguido identificar y analizar la racionalidad y los discursos legitimadores de la política cultural en torno a la película Vicky Cristina Barcelona. La mejora de la marca Barcelona, destinada a la atracción de turistas y a posibilitar externalidades económicas asociadas a esta industria, fue el objetivo prioritario del apoyo gubernamental al filme. Según el enfoque de la administración local, los procesos territoriales derivados de la película -como el aumento de visitantes a la ciudad-, se asociarían a la capacidad de city/destination branding del filme. Por lo tanto, su efectividad se ha vinculado a su calidad como "estrategia de comunicación”, articulada entre la productora Mediapro y el propio Ayuntamiento de Barcelona (González, 2009). En el marco de esta estrategia, la película ha sido considerada como un caso exitoso de creación de marca mediante un proyecto privado con financiación pública, señalándose asimismo que dicha contribución económica era reducida "si se compara con el presupuesto de una campaña de publicidad en medios internacionales y de gran impacto” (Marco, 2012, p. 198). Así, la película se presenta como un ejemplo de "publicidad cinematográfica", y la participación pública en su producción formó parte de una estrategia de place branding apoyada 
en la industria cultural y en el patrimonio urbano, particularmente el modernismo arquitectónico.

En cambio, la actuación del Gobierno catalán en este proyecto se orientó hacia el desarrollo de las industrias culturales, del campo artístico catalán, y buscó acompañar las políticas de promoción lingüística a nivel autonómico. No obstante, dicha estrategia se vio limitada en su capacidad de alcanzar sus objetivos y secundó la estrategia de marca. Por un lado, si bien el número de largometrajes producidos en Barcelona ha crecido desde el estreno de Vicky Cristina Barcelona, el llamado "efecto Woody Allen" no puede considerarse en sí mismo un salto cuantitativo relevante en este sentido para la ciudad o para Cataluńa. Por otro lado, la colaboración público-privada establecida para este proyecto derivó en una propuesta de difusión internacional de la ciudad, que proyecta una imagen de esta adaptada al consumidor anglosajón. Se trata de una narración sobre el entorno urbano que, como ya indicamos, presenta poca relación con la identidad local, con la lengua catalana o con las formas de vida en la ciudad. En este sentido, la marca se vería más bien reforzada por la asociación de la ciudad con su patrimonio característico, con el propio director cinematográfico y su prestigio internacional, así como con la ciudad de Nueva York.

De este modo, nuestro análisis del caso confirma nuestra hipótesis al revelar la instrumentalización, por parte de diferentes actores públicos y privados, de la producción audiovisual y del patrimonio cultural-arquitectónico de la ciudad para promover la marca ciudad. El proceso se figura como un ejemplo de la difuminación de las fronteras entre branding y política cultural, reflejado en las actuaciones correspondientes a las áreas de gobierno implicadas, aunque revelando algunas prioridades y agendas comunes a estos ámbitos de la acción pública. En este sentido, la acción pública analizada evidencia la importancia de la cultura como instrumento de marca y generación de prestigio asociado a la misma (Urry, 2002), y que la creciente internacionalización de las políticas culturales (Singh, 2010) tiene como base múltiples entendimientos de la gestión pública de la cultura en la economía actual (Zamorano, 2016).

Si bien este caso se inserta en una negociación política caracterizada por ciertos intereses convergentes y otros en tensión, sintetiza asimismo diversos efectos ya señalados para las políticas enmarcadas en el concepto de la ciudad creativa, como son la creciente mercantilización de la cultura y su inscripción en el modelo emprendedor de políticas culturales (Bustamante, 2011; Hutton, 2017). Este modelo ha promovido la "apertura" del campo cultural tradicional -incluidas las artes cultas y populares y la industria del cine- a la incorporación de nuevos sectores productivos pertenecientes a la llamada economía creativa, como el textil, el videojuego o el turismo cultural, y una mayor interrelación de dichos ámbitos con fines de eficiencia productiva. Como ya hemos señalado, la literatura ha indicado que dicho marco de apertura y a la vez (re)elitización cultural, conlleva el relegamiento de los fines redistributivos de capital cultural y de representación de las realidades culturales, con respecto a los fines de productividad y competitividad económica (Balibrea, 2017; Dinardi, 2017; Hutton, 2017). En esta línea, la estrategia estudiada posee un énfasis en las políticas de promoción del sector turístico mediante la creación 
de marca Barcelona, políticas que ya han sido relacionadas con la progresiva "tematización” del entorno urbano, la gentrificación y especulación inmobiliaria, y que también han sido caracterizadas por su ambigua relación con el sector cultural (Balibrea, 2005, 2017; Delgado, 2008, p. 2; Rius Ulldemolins, 2014).

El avance del branding cultural tiene, en consecuencia, diversas implicaciones para la orientación actual de la política cultural. La competencia en el mercado global por el posicionamiento de las marcas urbanas y la instrumentalización de la cultura y el patrimonio urbano con estos fines, se establecen en un diálogo continuo con los actores internacionales definidos como relevantes para la economía creativa, sus intereses y expectativas (Lury, 2004; Vicari Haddock, 2010), dado que la asociación del territorio a unos valores adaptados a las segmentadas expectativas de consumo externas, sería uno de los instrumentos para alcanzar un mejor posicionamiento en este mercado (Florida, 2002; Jones \& Smith, 2005). Pero, como evidencia el caso estudiado, la influencia de esta lógica en la construcción de las políticas culturales puede favorecer su relativo alejamiento de las necesidades sociales y sectoriales locales, así como distintas transformaciones en la realidad social representada, orientadas a reafirmar elementos simbólicos asociados a la marca (Palou Rubio, 2006, p. 24). En este sentido, la creación de marca de base cultural representa una de las características distintivas de la política cultural emprendedora en relación con los modelos de política cultural de orientación redistributiva y de promoción de las artes (Zimmer \& Toepler, 1999). Esto se manifiesta con particular claridad en el campo de las industrias culturales, un dominio que requiere de una elevada contribución pública.

Dicha transformación de las políticas culturales abarca también el rol de las industrias culturales como instrumentos de promoción y reproducción simbólica. La gestión pública de este sector cultural se puede ver marcada por mecanismos orientados a la simplificación de la realidad cultural representada y a la satisfacción de necesidades de consumo, indispensables para el desarrollo efectivo de las estrategias de marca. En este sentido, a inicios del siglo xx la crítica al control estatal sobre las industrias culturales se centró en sus potencialidades para el control de masas. Asimismo, destacó que una industria cultural liberada a las leyes del mercado podría contribuir a la trivialización del arte -en función de su estética hegemónica y reproductibilidad- (Horkheimer \& Adorno, 1988). Actualmente, la promoción pública de una representación creativa de las ciudades a nivel internacional, con base en la publicidad cinematográfica de su patrimonio y oferta cultural, se exhibe como un medio para la liberación individual, en tanto marca/discurso de consumo adaptado a cada ciudadano o turista. Así, sobre la base del gran capital simbólico asociado al consumo turístico en el mundo contemporáneo, el caso estudiado evidencia una racionalidad instrumental aplicada a la gestión pública de la cultura caracterizada por la búsqueda de competitividad simbólico-territorial. 


\section{Referencias bibliográficas}

Aertsen, V. (2011). El cine como inductor del turismo. La experiencia turística en Vicky, Cristina, Barcelona. Razón y palabra, 77, 53-78. http://www.razonypalabra.org.mx/ varia/77\%204a\%20parte/54_Aersten_V77.pdf

Agencias. (2007, julio 9). Woody Allen inicia con polémica en Barcelona el rodaje de su nueva película. El Mundo. https://www.elmundo.es/elmundo/2007/07/09/ cultura/1183966614.html

Anholt, S. (2003). Brand new justice: The upside of global branding. Oxford: ButterworthHeinemann.

Anholt, S. (2006). Competitive identity: The new brand management for nations, cities and regions. Basingstoke: Palgrave Macmillan.

Aronczyk, M. (2008). "Living the Brand": Nationality, globality and the identity strategies of nation branding consultants. International Journal of Communication, 2(1), 41-65. https://ijoc.org/index.php/ijoc/article/view/218/118

Bailey, P. J. (2001). The reluctant film Art of Woody Allen. Kentucky: The University Press of Kentucky.

Balibrea, M. P. (2005). Barcelona: del modelo a la marca. En J. Carrillo \& I. Estella Noriega (eds.), Desacuerdos 3. Sobre arte, politicas y esfera pública en el Estado español. Barcelona: Arteleku-Museo de Arte Contemporáneo de Barcelona (МАСвA)-Universidad Internacional de Andalucía.

Balibrea, M. P. (2017). The global cultural capital. Addressing the citizen and producing the city in Barcelona. London: Palgrave.

Bell, D. (1976). The cultural contradictions of capitalism. Portsmouth: Heinemann Educational.

Benach, N. \& Tello, R. (2004). En los intersticios de la renovación. Estrategias de transformación del espacio y flujos de población en Barcelona. Revista de Geografía, 3, 93-114. https:// www.raco.cat/index.php/RevistaGeografia/article/view/46143

Bianchini, F. (1993). Urban cultural Policy in Britain and Europe: towards cultural planning. London: Institute for Cultural Policy Studies.

Bourdieu, P. (2003). Un arte medio: ensayo sobre los usos sociales de la fotografía. Barcelona: Editorial Gustavo Gili.

Bustamante, E. (ed.). (2011). Industrias creativas. Amenazas sobre la cultura digital. Barcelona: Gedisa.

Cazorla, B. (2008, agosto 23). El Llull pagó 100.000 euros al Cervantes por las clases de catalán. El País.

Celis, B. (2007, marzo 7). Barcelona según Woody Allen. El cineasta neoyorquino tiene previsto rodar también en Oviedo su próximo filme. El Pais. https://elpais.com/ diario/2007/03/07/catalunya/1173233255_850215.html

Chen, F. \& Mele, C. (2016). Film-induced pilgrimage and contested heritage space in Taipei. City, Culture and Society, 9, 31-38. https://doi.org/10.1016/j.ccs.2016.02.001

Cox, R. (1999). Multilateralism and world order. En R. Cox \& T. J. Sinclair (eds.), Approaches to world order (pp. 494-523). Cambridge: Cambridge University Press.

Dargis, M. (2008, agosto 15). The portrait of two ladies. New York Times. https://www.nytimes. com/2008/08/15/movies/15barc.html 
De Rivera, C. (2009). El cine catalán o en catalán. Ciudadanos. Nota de Prensa. https://www. ciudadanoscs.org/prensa/Carmen_de_Rivera_El_cine_catalan_o_en_catalan/3068

Del Rey-Reguillo, A. (ed.). (2007). Cine, imaginario y turismo. Valencia: Tirant lo Blanch.

Delgado, M. (2008). La artistización de las políticas urbanas. El lugar de la cultura en las dinámicas de reapropiación capitalista de la ciudad. Scripta Nova. Revista Electrónica de Geografia y Ciencias Sociales, 12. http://www.ub.edu/geocrit/sn/sn-270/sn-270-69. htm

Departament de Cultura (2008). Figures for Catalonia. Barcelona: Generalitat de Catalunya.

DiGiacomo, S. (2008). "Vicky Cristina Barcelona” vista per una antropòloga. Quaderns-e de L'Institut Català d'Antropologia, 11.

Dinardi, C. (2017). Cities for sale: Contesting city branding and cultural policies in Buenos Aires. Urban Studies, 54(1), 85-101. https://doi.org/10.1177\%2F0042098015604079

Dinnie, K. (2008). Nation branding concepts, issues, practice. Oxford: Butterworth-Heinemann.

Evans, G. (2003). Hard-branding the cultural city-from Prado to Prada. International Journal of Urban and Regional Research, 27(2), 417-440. https://doi.org/10.1111/14682427.00455

Falicov, T. (2018). Diversifying public film funding policies in Latin America. En P. C. Murschetz, R. Teichman, \& M. Karmasin (eds.), Handbook of State Aid for Film (pp. 367-382). New York: Springer.

fapae (Confederación de Productores Audiovisuales Españoles). (2008). Memoria anual 2008. Madrid: FAPAE.

Florida, R. (2002). The rise of the creative class: and how it's transforming work, leisure, community and everyday life. New York: Basic Books.

Fresán, R. (2012, julio 8). Mediodía en Barcelona. Página 12. https://www.pagina12.com.ar/ diario/suplementos/radar/subnotas/8061-1752-2012-07-12.html

García, B. (2004). Urban regeneration, arts programming and major events: Glasgow 1990, Sydney 2000 and Barcelona 2004. International Journal of Cultural Policy, 10(1), 103118. https://doi.org/10.1080/1028663042000212355

Generalitat de Catalunya (2008). Comissió de Política Cultural. Sessió núm. 27 / Política Cultural / 10 de desembre de 2008 Fitxer 01. vin legislatura / Cinquè període / Dimecres, 10 de desembre.

Generalitat de Catalunya (2010). Diari Oicial de la Generalitat de Catalunya nom. 5628 13.5.2010. Смc/1480/2010, de 23 d'abril, per la qual es dóna publicitat a les subvencions concedides per l'Institut Català de les Indústries Culturals durant el primer semestre de l'any 2009.

González, J. T. (2009). La creatividad está en el ADN de Barcelona: el Ayuntamiento de Barcelona invierte 22 millones de euros anuales en comunicación. Ipmark, 16(720), 12-14.

Hanna, S. \& Rowley, J. (2008). An analysis of terminology use in place branding. Place Branding and Public Diplomacy, 4(1), 61-75. https://doi.org/10.1057/palgrave.pb.6000084

Harvey, D. (1989). From managerialism to entrepreneurialism: The transformation in urban governance in late capitalism. Geografiska Annaler. Series B, Human Geography, 71(1), 3-17. https://doi.org/10.1080/04353684.1989.11879583

Hernández R. J. (2004). Turismo inducido. La configuración de la imagen turística de Sevilla a través del cine comercial. En I Congreso Internacional Patrimonio, Desarrollo Rural y Turismo en el Siglo XXI, Osuna. 
Horkheimer, M. \& Adorno, T. (1988). Theodor, Dialéctica del iluminismo. Buenos Aires: Sudamericana.

Hudson, S. \& Brent Ritchie, J. R. (2006). Film tourism and destination marketing: the case of Captain Corelli's Mandolin. Journal of Vacation Marketing, 12(3), 256-268. https:// doi.org/10.1177\%2F1356766706064619

Hutton, T. (2017). The creative city. En J. Rennie Short (ed.), A research agenda for cities (pp.137-151). Cheltenham: Edward Elgar.

Institut Català de les Indústries Culturals (ICIC) (2012). Memòria anual del Institut Català de les Indústries Culturals. Barcelona: Generalitat de Catalunya.

Instituto de Cultura de Barcelona (ICUB). (2007). Barcelona Cultura 07 Balanç. Barcelona: Ajuntament de Barcelona.

Instituto de Cultura de Barcelona (ICUB). (2008). Memòria de l'Institut de Cultura de Barcelona. Barcelona: Ajuntament de Barcelona.

Instituto de Estadística de Cataluña (Idescat) (2010): Anuario estadístico de Cataluña. Cine. Películas, sesiones, espectadores y recaudación. Por nacionalidad de la película. http:// www.idescat.cat $/$ pub/?id=aec\&n=777\&lang=es\&t=2010

IMDb (Internet Movie Database. Box office / business for Vicky Cristina Barcelona, 2008. http://www.imdb.com/title/tt0497465/business.

Jessop, B. (2002). The future of the capitalist state. London: Polity.

Jones, D. \& Smith, K. (2005). Middle-earth meets New Zealand: Authenticity and location in the making of The Lord of the Rings. Journal of Management Studies, 42(5), 923-945. https://doi.org/10.1111/j.1467-6486.2005.00527.x

Landry, C. \& Bianchini, F. (1995). The creative city. London: Demos.

Leonard, M. (1997). Britain: renewing our identity. London: Demos.

Leonard, M. (2002). Public diplomacy. London: Foreign Policy Centre.

Lewis, N. (2011). Packaging political projects in geographical imaginaries: the rise of nation branding. En A. Pike (ed.), Brands and branding geographies (pp. 264-287). Cheltenham, uk: Edward Elgar Publishing. https://doi.org/10.4337/9780857930842.00026

Lury, C. (2004). Brands. The logos of the global economy. London: Routledge.

Majoor, S. (2011). Framing large-scale projects: Barcelona forum and the challenge of balancing local and global needs. Journal of Planning Education and Research, 31(143), 143-156. https://doi.org/10.1177\%2F0739456X11402694

Marco, E. (2012). La cultura en la esencia de la Marca España: nuevos retos y estrategias para la proyección exterior. En Escuela Diplomática. Retos de nuestra acción exterior: Diplomacia Pública y Marca España (pp.193-201). Madrid: Ministerio de Asuntos Exteriores.

Mark, S. (2008). A comparative study of the cultural diplomacy of Canada, New Zealand and India. Tesis doctoral. University of Auckland, Auckland, Nueva Zelanda. https:// researchspace.auckland.ac.nz/bitstream/handle/2292/2943/02 whole.pdf?sequence=9

Martínez Expósito, A. (2014). La marca Asturias en el cine español: de Volver a empezar a Vicky Cristina Barcelona. En S. Bando \& M. Insúa (eds.), Actas del II Congreso Ibero-Asiático de Hispanistas (Kioto, 2013) (pp.341-355). Pamplona: Servicio de Publicaciones de la Universidad de Navarra. http://dadun.unav.edu/handle/10171/37163

Menchén, M. (2011, 4 de julio). Mediapro hace su agosto particular con Woody Allen. Expansión. http://www.expansion.com/2011/07/04/catalunya/1309746896.html 
Menger, P. (2010). Cultural policies in Europe. From a state to a city-centered perspective on cultural generativity. GRIPS Discussion Paper 10-28. Tokyo: National Graduate Institute for Policy Studies. http://www3.grips.ac.jp/ - pinc/data/10-28.pdf

Ministerio de Cultura, España (2008). Conferencia Sectorial de Cultura. Memoria de Actividades 2007. Madrid: Ministerio de Cultura.

Moilanen, T. \& Rainisto, S. (2008). How to brand nations, cities and destinations: A planning book for place branding. New York: Palgrave McMillan.

Montalt, S. (2007, mayo 18). Allen posa Catalunya en el punt de mira. Diari de Girona. http:// www.diaridegirona.cat/cultura/2008/05/18/allen-posa-catalunyapunt-mira/266407. html

Moragas i Spa, M. (2009). Informe de la comunicació a Catalunya 2007-2008. Barcelona: Institut de la Comunicació, Universitat Autònoma de Barcelona (InCom-UAB) / Generalitat de Catalunya.

Négrier, E. (1997). French cultural decentralization and international expansion. Towards a geometrically variable interculturalism? International Journal of Urban and Regional Research, 21(1), 63-74. https://doi.org/10.1111/1468-2427.00057

Opello, W. C. \& Rosow, S. J. (1999). The Nation-State and global order: A historical introduction to contemporary politics. Boulder, co: Linne Rienner.

Palou Rubio, S. (2006). La ciudad fingida. Representaciones y memorias de la Barcelona turística. Pasos: Revista de Turismo y Patrimonio Cultural, 4(1), 13-28. https://doi. org/10.25145/j.pasos.2006.04.002

Parlament de Catalunya (2008). Butlletí oficial, viı legislatura, Número 270, Quart período, Dimarts, 27 de maig de 2008.

Peck, J. (2005). Struggling with the Creative Class. International Journal of Urban and Regional Research, 29(4), 740-770. https://doi.org/10.1111/j.1468-2427.2005.00620.x

Peters, G. (1995). Modelos alternativos del proceso de la política pública. De abajo hacia arriba o de arriba hacia abajo. Gestión y Política Pública, 4(2), 257-276. http://www. gestionypoliticapublica.cide.edu/?page_id=289

Pingree, G. (2007, 27 de julio). Woody Allen's Barcelona Problem, TIME. http://content.time. com/time/arts/article/0,8599,1648406,00.html

Ren, C. \& Blichfeldt, B. S. (2011). One clear image? Challenging simplicity in place branding, Scandinavian Journal of Hospitality and Tourism, 11(4), 416-434. https://doi.org/10.1 080/15022250.2011.598753

Rius Ulldemolins, J. (2014). Culture and authenticity in urban regeneration processes: Place branding in central Barcelona. Urban Studies, 51(14), 3026-3045. https://doi. org/10.1177\%2F0042098013515762

Rius Ulldemolins, J. \& Zamorano, M. M. (2015). Spain's nation branding project Marca España and its cultural policy: the economic and political instrumentalization of a homogeneous and simplified cultural image. International Journal of Cultural Policies, 21(1), 20-40. https://doi.org/10.1080/10286632.2013.877456

Rodríguez Campo, L., Fraiz Brea, J. A., \& Rodríguez-Toubes Muñiz, D. (2011). Tourist destination image formed by the cinema: Barcelona positioning through the feature film Vicky Cristina Barcelona. European Journal of Tourism, Hospitality and Recreation, 2(1), 137-154. https://bit.ly/2lENPNM

Rodríguez Morató, A. (ed.). (2007). La sociedad de la cultura. Barcelona: Ariel. 
Savall, C. (2009, enero 26). La Generalitat activa un plan de choque para salvar al cine catalán. El Periódico. Reproducido en Alternativa Ciudadana Progresista.http://www. alternativaciudadana.es/2009/01/26/el-perio-15/

Schneider, A. \& Ingram, H. (1997). Policy design for democracy. Lawrence, ks: University of Kansas Press.

Scott, A. (2010). Cultural economy and the creative field of the city. Geografiska Annaler: Series B, Human Geography, 92(2), 115-130. https://doi.org/10.1111/j.14680467.2010.00337.x

Singh, J. P. (2010). International cultural policies and power. New York: Palgrave Macmillan.

Stanishevski, K. (2007). La comunicación de los destinos turísticos. En A. del Rey-Reguillo (ed.), Cine, imaginario y turismo. Estrategias de seducción (pp. 245-265). Valencia: Tirant lo Blanch.

Turisme de Barcelona (2010). Estadistiques de turisme a Barcelona. Barcelona: Turisme de Barcelona.

Urry, J. (2002). The tourist gaze. London: Sage.

Vanolo, A. (2008). The image of the creative city: Some reflections on urban branding in Turin. Cities, 25(6), 370-382. https://doi.org/10.1016/j.cities.2008.08.001

Veblen, T. (1994). The theory of the leisure class. Harmondsworth: Penguin.

Vicari Haddock, S. (2010). Brand-building: The creative city: a critical look at current concepts and practices. Firenze: Firenze University Press.

Villanueva, C. (2007). Representing cultural diplomacy: soft power, cosmopolitan constructivism and nation branding in Mexico and Sweden. Sweden: Växjö University Press.

Volkerling, M. (2001). From cool Britannia to hot nation: 'creative industries' policies in Europe, Canada and New Zealand. International Journal of Cultural Policy, 7(3), 437455. https://doi.org/10.1080/10286630109358155

Widler, J. (2007). Nation branding: with pride against prejudice. Place Branding and Public Diplomacy, 3(2), 144-150. https://doi.org/10.1057/palgrave.pb.6000055

Zamorano, M. M. (2016). Reframing cultural diplomacy: the instrumentalization of culture under the soft power theory. Culture Unbound, 8, 166-186. https://doi.org/10.3384/ cu.2000.1525.1608165

Zamorano, M. M. \& Rodríguez Morató, A. (2015). The cultural paradiplomacy of Barcelona since the 1980s: understanding transformations in local cultural paradiplomacy. International Journal of Cultural Policy, 21(5), 554-576. https://doi.org/10.1080/10 286632.2014.943752

Zimmer, A. \& Toepler, S. (1999). Cultural policies and the Welfare State: the cases of Sweden, Germany, and the United States. The Journal of Arts Management, Law and Society, 26, 167-193. https://doi.org/10.1080/10632921.1996.9942961

Zukin, S. (1996). Space and symbols in an age of decline. En A. King (ed.), Re-presenting the city (pp. 43-59). London: Macmillan. 


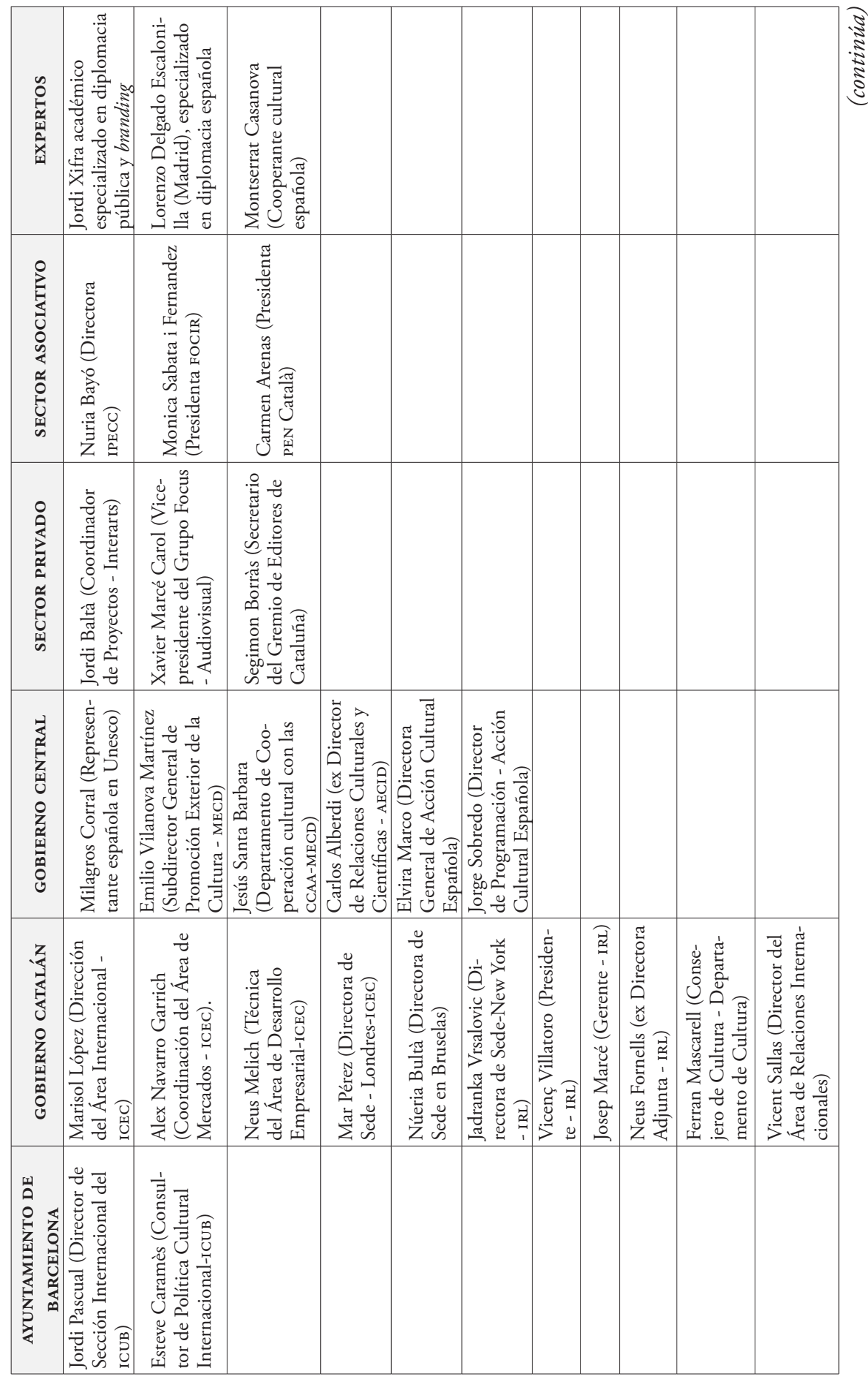




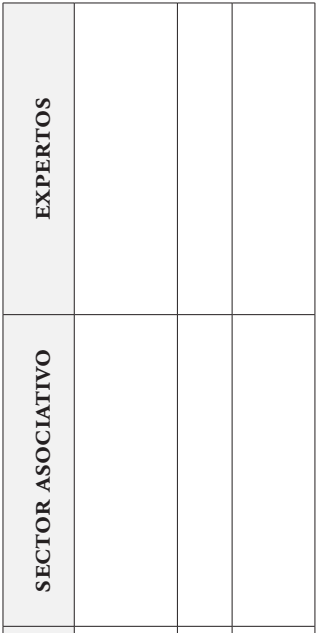

ż讠

获음

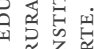

涫先。

엄

总界界

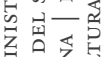

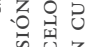

t苗

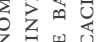

田

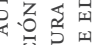

出虽

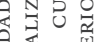

质畧思

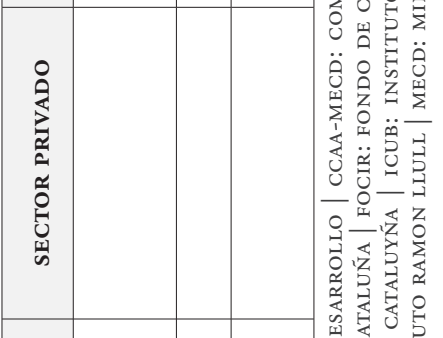

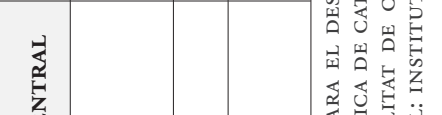

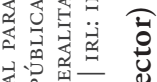

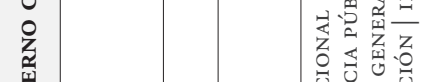

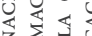

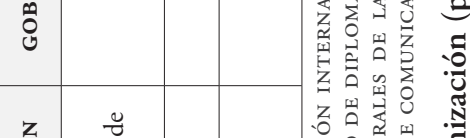

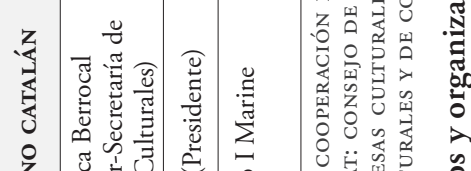

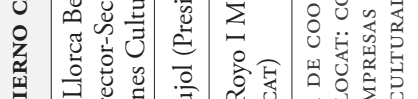

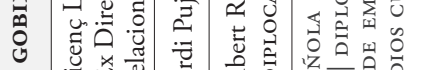

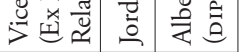

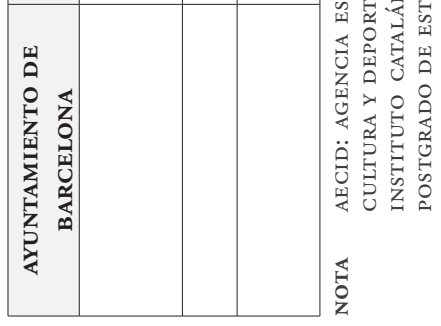

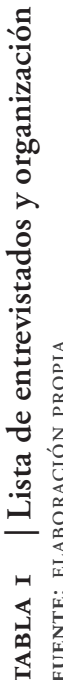

